\title{
Ponatinib Regimen
}

National Cancer Institute

\section{Source}

National Cancer Institute. Ponatinib Regimen. NCI Thesaurus. Code C160111.

A chemotherapy regimen consisting of ponatinib that may be used in the treatment of chronic myeloid leukemia (CML) and Philadelphia chromosome-positive acute lymphoblastic leukemia (ALL). 